\title{
Introduction to the special section on kidney transplantation
}

\author{
Ghaneh Fananapazir $^{1}$ \\ ${ }^{1}$ University of California Davis Medical Center, Sacramento, CA, USA
}

Kidney transplantation is the treatment of choice for most patients with end-stage kidney disease, and imparts better quality of life, lower risk of death, and lower treatment cost when compared with dialysis. Each year in the United States, over 100,000 people are diagnosed with end-stage kidney disease; however, the number of kidney grafts available is limited, and the number of kidney transplants performed each year hovers around 30,000 . Therefore, careful screening of potential donors and recipients and optimizing management of posttransplantation is important. Imaging plays a key role in both the potential donor and recipient and in identifying post-transplant complications. Interpretation of imaging requires an understanding of surgical considerations related to technique as well as the kidney graft's common and unique pathologies compared with native kidneys.

In this special section in Abdominal Radiology on kidney transplantation, we present reviews on disease processes, how to optimize existing protocols (ultrasound, CT, and MRI), and explore innovations and areas of active research that exist in certain modalities.

Our special section leads with understanding the kidney graft's unique and common disease processes, divided into two parts: one, by Fananapazir et al., highlights vascular complications of kidney grafts, and another, by Kim et al., looks at the nonvascular complications.

Articles contributed by Galgano et al., Marin et al., and Gondalia et al. present information on optimizing the existing modalities of ultrasound, CT, and MRI, respectively, in diagnostic performance. An article by Patel et al. provides an excellent overview of how to optimize success in the performance of ultrasound-guided kidney graft biopsies.

Two articles by Morgan et al. and Ljimani et al. highlight areas of active research in ultrasound and MRI, respectively. Particularly with MRI, functional evaluation of kidney grafts has shown promise in opening new avenues in diagnosing pathology. It is hoped that these articles will provide fodder for future research endeavors.

My gratitude is extended towards all the authors who contributed articles towards this special section on kidney transplantation. The work presented here provides a comprehensive overview of imaging of kidney grafts as well as areas of active research. I hope that you will find this to be a valuable resource. 